Geopolítica(s) Revista de estudios sobre espacio y poder ISSN: 2172-3958

\title{
Geopolítica de la Amazonia ${ }^{1}$
}

\section{Bertha Koiffmann Becker}

Resumen. La Amazonia ha sufrido profundos cambios estructurales. Nuevos actores tienen hoy un papel decisivo: la sociedad civil organizada, los gobiernos estatales y la cooperación internacional. Estos cambios se perciben según los diversos intereses dominantes en la escala global, nacional y regional, generando conflictos que dificultan la implementación de políticas públicas adecuadas. La Amazonía es hoy una región en sí, que demanda una política de consolidación del desarrollo. El fortalecimiento institucional, la Ciencia, Tecnología \& Innovación y la regionalización son estrategias sugeridas para ese fin.

Palabras clave: Amazonia; Brasil; geopolítica; globalización; desarrollo sostenible.

\section{[en] Geopolitics of Amazonia}

\begin{abstract}
Intense structural changes have occurred in the Amazon region. New actors have a determinant role: organized civil society, provincial governments and international cooperation. Changes are perceived according to different interests at the global, national and regional scales, resulting in conflicts that turn it difficult to implement public policies. Today, the Amazon is not a mere frontier, but a region by itself, that demands a development consolidation policy. Strategies to attain this goal are: institutional enforcement, Science, Technology \&Innovation and regionalization.
\end{abstract}

Keywords: Amazon; Brazil; geopolitics; globalization; regional development.

\section{[pt] Geopolítica da Amazônia}

Resumo. Profundas mudanças estruturais ocorreram na Amazônia onde novos atores têm hoje papel decisivo: a sociedade civil organizada, os governos estaduais e a cooperação internacional. Tais mudanças são percebidas segundo diversos interesses dominantes na escala global, nacional e regional, gerando conflitos que dificultam a implementação de políticas públicas adequadas. A Amazônia é hoje uma região em si, que demanda uma política de consolidação do desenvolvimento. Fortalecimento institucional, Ciência, Tecnologia \& Inovação e regionalização são estratégias sugeridas para esse fim.

Palavras-chave: Amazônia; Brasil; geopolítica; globalização; desenvolvimento sustentável.

Sumario. Introducción. 1. La Amazonia y la mercantilización de la naturaleza. 1.1. La dinámica regional reciente. 1.2. Globalización y Amazonia como frontera del capital natural. 1.3. La integra-

1 (Nota de la traducción) Agradecemos a los editores de la revista Estudos Avançados y a Lidia Becker y Beatriz Becker por la autorización de esta traducción El texto original, "Geopolítica da Amazônia", fue publicado por Estudos Avançados, vol. 19, núm. 53, 2005, 71-86. Traducido por Flávia Melo (Universidade de São Paulo) y revisado por María Lois (Universidad Complutense de Madrid). 
ción de la Amazonía sudamericana. 2. La Amazonia en el espacio nacional: una región en sí. 2.1. Las nuevas facciones de la frontera móvil. 2.2. La consolidación del poblamiento. Para terminar: ¿Cómo impedir la destrucción de los bosques? El papel de las políticas públicas.

Cómo citar: Becker, Bertha K. (2019) "Geopolítica de la Amazonia”. Geopolítica(s). Revista de estudios sobre espacio y poder, vol. 10, núm. 1, 135-151.

\section{Introducción}

En principio, cabe una pequeña explicación sobre geopolítica: se trata de un campo de conocimiento que analiza relaciones entre poder y espacio geográfico. Fue el fundamento del poblamiento de la Amazonia, desde el tiempo colonial, una vez que, por más que quisiera la Corona, no tenía recursos económicos y población para poblar y ocupar un territorio de tal extensión. Portugal logró conservar la Amazonia y expandirla más allá de los límites establecidos en el Tratado de Tordesillas gracias a estrategias de control sobre el territorio. Aunque los intereses económicos prevalecieron, no tuvieron éxito, y la geopolítica fue más importante que la economía en el sentido de garantizar la soberanía sobre la Amazonia, cuya ocupación se hizo, como se sabe, en picos vinculados a demandas externas sucedidos por extensos periodos de estancamiento y de decadencia.

La geopolítica siempre se caracterizó por la presencia de presiones de todo tipo, y por intervenciones en el escenario internacional desde las más blandas hasta incluso guerras y conquistas de territorios. Inicialmente, esas acciones tenían como sujeto fundamental el Estado entendido como la única fuente de poder, la única representación de la política, y las diferentes disputas eran analizadas solo entre Estados. Hoy, esta geopolítica actúa, sobre todo, a través del poder para influir en la toma de decisiones de los Estados sobre el uso del territorio, una vez que la conquista de territorios y colonias se han vuelto muy caras.

Así, se verificaría el fortalecimiento de lo que se denomina coerción velada. Presiones de todo tipo para influir en la decisión de los Estados sobre el uso de sus territorios. Este cambio está intrínsecamente vinculado a la revolución científicotecnológica y a las posibilidades de ampliar la comunicación y la circulación en el planeta a través de flujos y redes que aceleran el tiempo y amplían las escalas de comunicación y de relaciones, configurando espacios-tiempos diferenciados.

El espacio siempre se ha asociado al tiempo. Y hoy, en la intensificación de diferentes espacios-tiempos reside una de las raíces de la geopolítica contemporánea. Las redes se desarrollan en los países ricos, en los centros del poder, donde el avance tecnológico es mayor y la circulación planetaria permite que se seleccionen territorios para inversiones, selección que depende de las potencialidades de los propios territorios. Por eso, al expandirse y sostener los recursos móviles (financieros e informacionales), las redes se socializan. Y esa socialización está generando movimientos sociales importantes, que también tienden a transnacionalizarse.

Hay hoy, por tanto, dos movimientos internacionales: uno a nivel del sistema financiero, de la información, del dominio del poder de las potencias; y otro en la tendencia al internacionalismo de los movimientos sociales. Todos los agentes sociales organizados, corporaciones, organizaciones religiosas, movimientos sociales, etc. tienen sus propias territorialidades, por encima y por debajo de la escala 
del Estado, sus propias geopolíticas, y tienden a articularse, configurando una situación mundial bastante compleja.

La Amazonia es un ejemplo vivo de esta nueva geopolítica, pues en ella se encuentran todos esos elementos. Es un desafío para el presente, no para el futuro. ¿Cuál es este desafío actual? La Amazonia, Brasil, y los demás países latinoamericanos son las antiguas periferias del sistema mundial capitalista. Su poblamiento y desarrollo fueron fundados de acuerdo con el paradigma de relación sociedadnaturaleza, que Kenneth Boulding denomina economía de frontera, y que significa que el crecimiento económico es visto como lineal e infinito y basado en la continua incorporación de tierra y de recursos naturales, que también se perciben como infinitos. Este paradigma de la economía de frontera realmente caracteriza toda la formación latinoamericana.

Hoy, es imperativo modificar ese patrón de desarrollo que alcanzó el auge en las décadas de 1960 a 1980. Es imperativo el uso no predatorio de las fabulosas riquezas naturales que la Amazonia contiene y también del saber de sus poblaciones tradicionales que poseen un conocimiento secular acumulado para tratar con el trópico húmedo. Esta riqueza tendría que ser mejor utilizada, y acabar con ese patrón de economía de frontera es un imperativo internacional, nacional y también regional. Ya hay en la región resistencias a la apropiación indiscriminada de sus recursos y actores que luchan por sus derechos. Este es un hecho nuevo porque, hasta entonces, las fuerzas exógenas ocupaban la región libremente, aunque con serios conflictos. Esta es una de las hipótesis de este texto.

Con las resistencias regionales los conflictos en la región alcanzan un nivel más elevado. No se trata ya sólo de un conflicto por la tierra, es el conflicto de una región con relación a las demandas externas. Estos conflictos de interés, así como las acciones que resultan de ellos contribuyen a mantener imágenes obsoletas sobre la región, dificultando la elaboración de políticas públicas adecuadas a su desarrollo.

Para que se pueda cambiar ese patrón de desarrollo es necesario entender los diferentes proyectos geopolíticos y sus actores, que están en la base de los conflictos, e intentar encontrar modos de compatibilizar el crecimiento económico con la conservación de los recursos naturales y la inclusión social. En fin, no se trata de mero ambientalismo, ni de otro momento destructivo más.

¿Cómo efectuar dicha compatibilización? Este es un gran desafío para la Ciencia y la Tecnología, y se señalarán aquí problemas en que la Ciencia puede aportar por medio de tres hipótesis:

1) el nuevo significado geopolítico de la Amazonía a nivel global como la gran frontera del capital natural;

2) el nuevo lugar de la Amazonia en Brasil, y

3) la urgencia de una nueva política de desarrollo y de estrategias básicas para implementarla.

\section{La Amazonia y la mercantilización de la naturaleza}

El punto de partida para hacer este análisis es el reconocimiento de profundos cambios estructurales que ocurrieron en la Amazonia en las últimas décadas del 
siglo XX. Sabemos que el proyecto de integración nacional acarreó perversidades en términos ambientales y sociales. Pero con sangre, sudor y lágrimas se debe reconocer lo que queda de positivo de ese proceso, porque son elementos con los que la región cuenta hoy para su desarrollo. Y no se pueden olvidar.

\subsection{La dinámica regional reciente}

A finales del siglo XX, hubo impactos negativos, pero también cambios estructurales y nuevas realidades generadas en la frontera, la cual tomo como espacio no plenamente estructurado y por eso mismo capaz de generar realidades nuevas. Entre los cambios, destaca la conectividad regional, uno de los elementos más importantes en la Amazonia. No se trata sólo de las carreteras, elementos que contribuyeron a la depredación de los recursos y de la sociedad, sino, sobre todo, de las telecomunicaciones, porque la red de telecomunicaciones en la Amazonía permitió articulaciones locales/nacionales, así como locales/globales. Otro cambio importante es la de la economía, que pasó de la exclusividad del extractivismo a la industrialización, con la explotación mineral y con la Zona Franca de Manaus ${ }^{2}$, que fue un puesto geopolítico avanzado colocado por el Estado en la frontera norte, en pleno ambiente extractivista tradicional. Hay problemas en la Zona Franca, pero hoy es gran productora no sólo de bienes de consumo duraderos, sino también de bienes industriales de dos ruedas, de telefonía e incluso de biotecnología.

Una gran modificación estructural ha tenido lugar en el poblamiento regional que se asentó a lo largo de las carreteras - y no a lo largo de la red fluvial, como en el pasado- y en el crecimiento demográfico, sobre todo urbano. Se generó en la región una movilidad espacial problemática, con fuerte migración y continua expropiación de la tierra y, así, ligada a un proceso de urbanización. A la vista de todo esto, la Amazonía registró la mayor tasa de crecimiento urbano en el país en las últimas décadas. En el censo de 2000 , el $70 \%$ de la población en la región Norte estaba ubicada en núcleos urbanos, aunque carentes de servicios básicos (Figura 1). Muchos no comparten esta tesis, porque no consideran tales núcleos como núcleos urbanos. Pero ese es el modelo de urbanización en Brasil y, además, la urbanización no se mide sólo por el crecimiento y el surgimiento de nuevas ciudades, sino también por la difusión de los valores de la urbanización para la sociedad. Por esa razón, desde la década de 1980, llamo a la Amazonía "bosque urbanizado".

Por otro lado, se organizó la sociedad de forma nunca vista. Los grandes conflictos de tierras y de territorios de 1960 a 1980 constituyeron un aprendizaje político y en los 1990 se transformaron en proyectos alternativos, sobre la base de la organización de la sociedad civil. Es extremadamente importante recordar que hoy, esa sociedad tiene voz activa en la Amazonia y en Brasil, incluyendo muchos grupos indígenas. Esta organización de la sociedad política trae, a su vez, cambios en la ocupación del territorio, con la multiplicación de unidades de conservación federales y estatales, así como también con la demarcación de tierras indígenas.

2 (Nota de la traducción) De acuerdo con la ley de su creación, la Zona Franca de Manaus es "un área de libre comercio de importación y exportación y de incentivos fiscales especiales, establecida con la finalidad de crear en el interior de la Amazonia un centro industrial, comercial y agropecuario dotado de condiciones económicas que permitan su desarrollo, frente a los factores locales y de la gran distancia a los que se encuentran, los centros consumidores de sus productos" (Brasil, Decreto Ley 288/1967). 


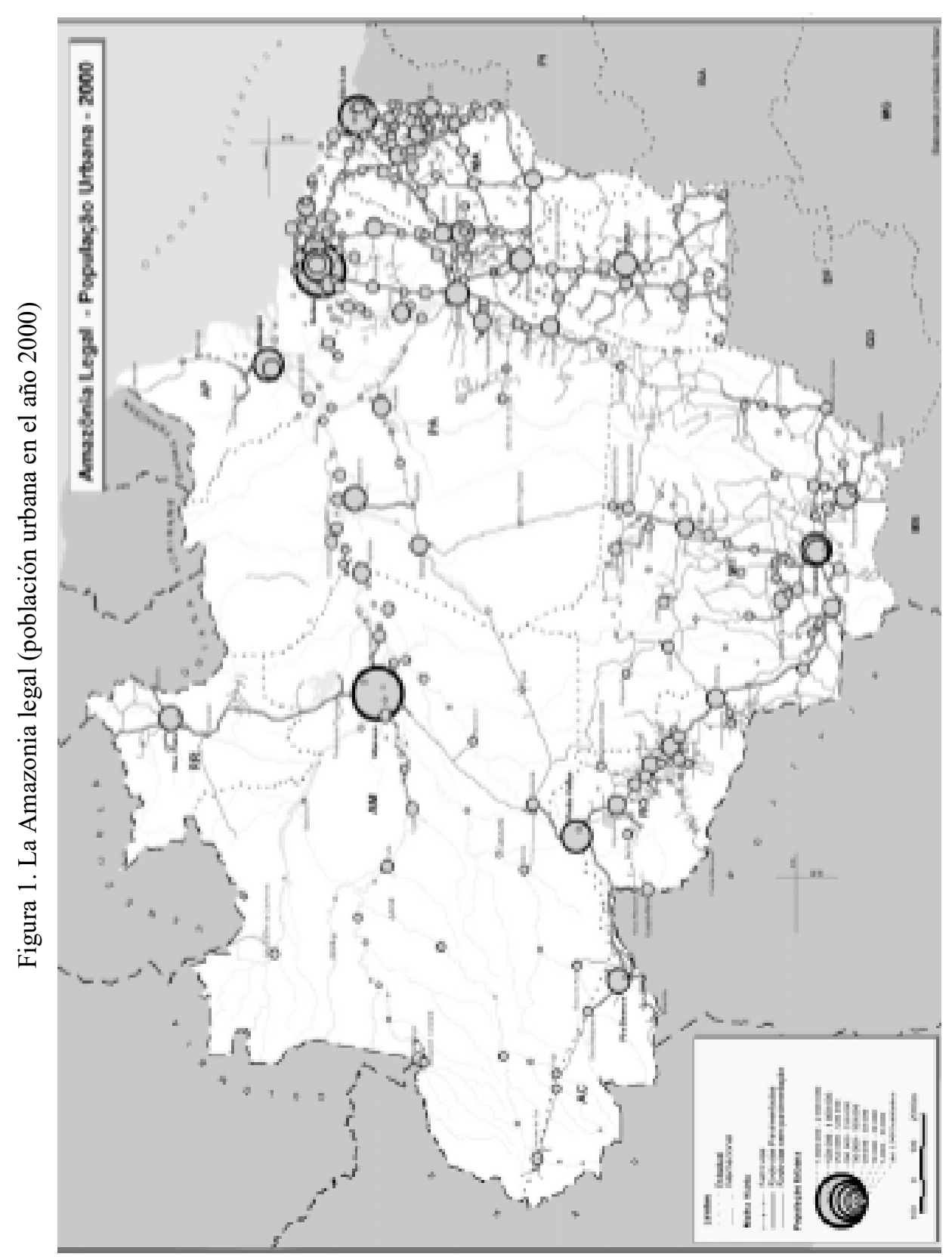

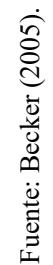


¿Qué proyectos y qué actores producen hoy la dinámica regional y los nuevos significados de la Amazonia? Estas transformaciones no son vistas de forma homogénea por los diferentes actores, porque dependen de intereses diversos y generan acciones diferentes en la región. Hay muchos conflictos dentro de esas percepciones, pero hay algunas dominantes.

El uso del método geográfico para el análisis de los proyectos geopolíticos y sus actores en diferentes escalas geográficas será útil en este análisis.

\subsection{Globalización y Amazonia como frontera del capital natural}

La primera hipótesis es la constitución de la Amazonía como frontera del capital natural a nivel global, en la que se identifican dos proyectos: el primero es un proyecto internacional para la Amazonia, y el segundo es el de la integración de la región Amazonia, sudamericana, continental.

Hasta hace poco, en el proyecto internacional dominaba la percepción de la Amazonia como una inmensa unidad de conservación a preservar, teniendo en vista la supervivencia del planeta, debido a los efectos de la deforestación sobre el clima y la biodiversidad. La base de esta percepción tuvo como origen, en gran parte, la tecnología de los satélites, que permitió por primera vez una visión de conjunto de la superficie de la Tierra y de su unidad trayendo el sentimiento de la responsabilidad común, así como la percepción del agotamiento de la naturaleza, que se ha convertido en un recurso escaso (Figura 2).

La naturaleza fue entonces reevaluada y revalorizada a partir de dos lógicas muy diferentes, pero que convergen para el mismo proyecto de preservación de la Amazonia. La primera lógica es la civilizatoria o cultural, que tiene una preocupación legítima con la naturaleza por la cuestión de la vida, lo que da origen a los movimientos ambientalistas. La otra lógica es la de la acumulación, que ve la naturaleza como recurso escaso y como reserva de valor para la realización de capital futuro, fundamentalmente en lo que se refiere al uso de la biodiversidad condicionada al avance de la tecnología. Otro recurso de que poco se habla, pero que ya es fundamental, es el agua como fuente de vida y de energía debido a los isótopos de hidrógeno, cuestión teórica aún no solucionada, pero que viene siendo investigada en muchos países, especialmente en Alemania y en Estados Unidos.

Se hace patente que, si hay una valorización de la naturaleza y de la Amazonia, hay también la relativización del poder de la virtualidad de los flujos y redes del mundo contemporáneo, con la globalización, que acabaría con las fronteras y con los Estados. En realidad, los flujos y redes no eliminan el valor estratégico de la riqueza localizada in situ: sostienen la riqueza circulante del sistema financiero, de la información, pero la riqueza localizada en el territorio también tiene su papel y su valor.

Consecuentemente, esto trajo una disputa de las potencias por las riquezas naturales, una vez que la distribución geográfica de tecnología y de recursos es desigual. Mientras que las tecnologías avanzadas se desarrollan en los centros de poder, las reservas naturales se localizan en los países periféricos, o en áreas no reguladas jurídicamente. Esta es, pues, la base de la disputa. 
Figura 2. Mapa de la vegetación de América del Sur

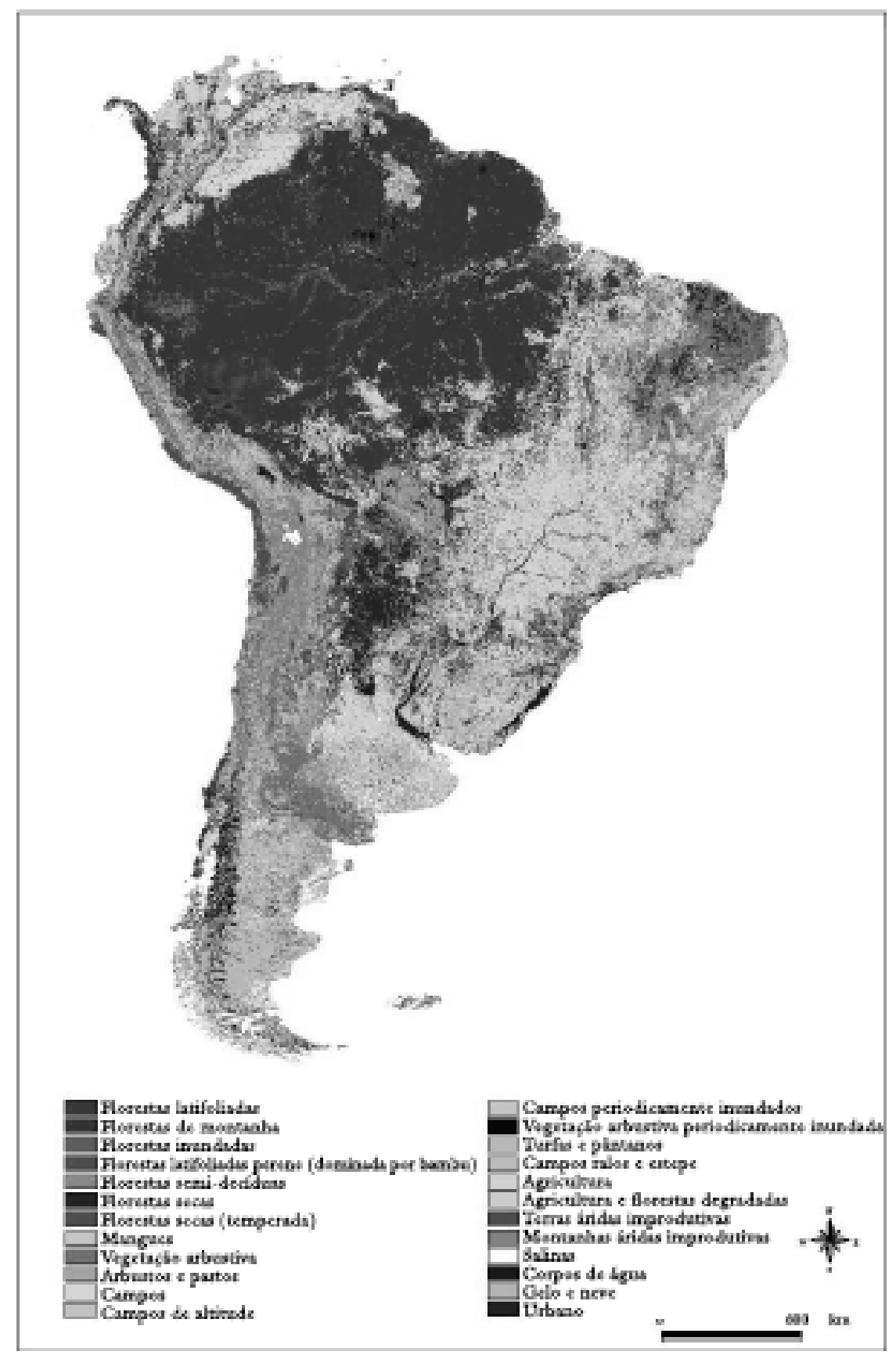

Fuente: Becker (2005). 
Hay tres grandes "el Dorados" naturales en el mundo contemporáneo: la Antártida, que es un espacio dividido entre las grandes potencias; los fondos marinos, riquísimos en minerales y vegetales, que son espacios no regulados jurídicamente; y la Amazonia, región que está bajo la soberanía de estados nacionales, entre ellos Brasil.

Este contexto geopolítico, principalmente en la década de 1980 y 1990, generó propuestas mundiales por una soberanía compartida y por poder administrar la Amazonia, que afectó incluso al Derecho Internacional. Hoy, sin embargo, los intereses ligados a la valorización del capital natural, que tiende a superponerse a la lógica cultural, van en aumento.

Observamos un proceso de mercantilización de la naturaleza. Los elementos de la naturaleza se están transformando en mercancías ficticias, usando la expresión de Karl Polanyi, en su libro La gran transformación. ¿Ficticias por qué? Porque no fueron producidas para la venta en el mercado (el aire, el agua, la biodiversidad). Pero, sin embargo, a través de esta ficción se generan mercados reales y esto se dio, como Polanyi muestra muy bien, al inicio de la industrialización, cuando tierra, dinero y trabajo se transformaron en mercancías ficticias, generando mercados reales.

¿Qué es el protocolo de Kyoto si no el mercado del aire? Es el intento de establecer cuotas de emisión de carbono en los países fuertemente industrializados y contaminantes a cambio del mantenimiento de bosques en los países que los tienen. El mercado del aire es el más avanzado. En otras palabras, esos mercados reales intentan institucionalizarse en foros globales, lo que también es una vertiente nueva dentro del Derecho Internacional.

No es ninguna fantasía el hecho de que lo que está en curso en la Amazonía es la transformación de bienes de la naturaleza en mercancías. Es el caso de Peugeot, que hace inversiones hacia el secuestro de carbono en Mato Grosso; en la isla de Bananal, la empresa inglesa S. Barry; la Maderera Mil, que tiene un proyecto en este sentido en el estado de Amazonas; la Central South West Corporations de Dallas, una empresa de energía que hizo una adquisición en Paraná de setecientas mil hectáreas de la reserva de la Serra de Itaqui con la mediación de la National Conservancy,; además de los proyectos que no conocemos, ya que unos son oficiales y otros no. Habría que hacer restricciones en ese sentido porque la tierra y el bosque son bienes públicos, y la venta de bosque significa venta de territorio y no es lo correcto desde el punto de vista del país.

Es digno de recordar que existen esfuerzos para regular el mercado de la biodiversidad, como el Prototipe Carbon Fund, del Banco Mundial, aunque es un sistema difícil de implementar ya que las patentes y la distribución de beneficios para las poblaciones locales no se han regulado en el país.

El mercado de los recursos hídricos es el más atrasado, aunque hay múltiples intentos de regularización de ese mercado. El agua se considera el oro azul del siglo XXI, en términos globales, porque hay escasez y consumo creciente en el mundo, sobre todo en los países semiáridos que utilizan la irrigación. Además, hay previsiones de que la disputa por agua puede llegar hasta conflictos armados.

Polanyi muestra que hay una necesidad de organización de la sociedad para impedir el libre juego de las fuerzas del mercado con relación a los elementos vitales para el hombre. Por ello se crearon sindicatos para proteger el mercado laboral y se 
organizaron asociaciones para regular el mercado de la tierra y el papel del estado se volvió fundamental. ¿Qué vamos a hacer en Brasil, y cuál debe ser hoy nuestra reacción? Tenemos todas esas bazas: bosques, biodiversidad, agua. ¿Cómo deben comportarse la sociedad y el gobierno brasileño con relación a su uso? Hoy, el movimiento de mercantilización es irreversible y tenemos que saber cómo lidiar con él. Me parece que cabría tanto que gobierno como sociedad luchasen por la regulación de esos mercados, pero debería ser bien negociada.

¿Cuáles son los principales actores en este proyecto internacional? Los movimientos ambientalistas, donde se destacan las ONG nacionales e internacionales, la cooperación internacional técnica, financiera, científica en grandes proyectos, como es el caso del Programa Piloto para la Protección de los Bosques Tropicales Brasileños (PPG7), del LBA y del Probem $1^{3}$, organizaciones religiosas de todo tipo, así como agencias de desarrollo de gobiernos extranjeros y también de empresas orientadas hacia el secuestro de carbono y/o madera certificada.

La cooperación internacional es fundamental para el desarrollo de la Ciencia y la Tecnología en Brasil. Pero, a veces, esa cooperación tecnocientífica tiene un exceso de autonomía. La cuestión crucial es el control de la información, porque muchas veces los investigadores brasileños, en alianzas, conocen el subproyecto ligado a su asociación, pero no el proyecto como un todo. Debe haber, por lo tanto, una concientización de los investigadores en el sentido de la globalización de la investigación, de modo que no tengan acceso sólo a una parte de la información.

La cooperación internacional tiene un lado extremadamente importante que es el de la relación con las comunidades locales, gracias a las redes de telecomunicación. Hay una fuerte presencia internacional que se estableció en la Amazonia debido también a los impactos del proyecto anterior, que excluía a las poblaciones regionales, expulsaba a pequeños productores y amenazaba a indios, y esos grupos consiguieron apoyos internacionales, como fue el caso de Chico Mendes ${ }^{4}$, que fue a Washington para obtener un refuerzo para mantener su propia supervivencia. Es necesario que la sociedad y el gobierno estén atentos a la cuestión de la soberanía interna, en el sentido de reconocer que el pueblo no es homogéneo y que tiene demandas diferentes que no son debidamente atendidas, lo cual genera conflictos que afectan la gobernabilidad.

\subsection{La integración de la Amazonía sudamericana}

Un segundo proyecto internacional se refiere a la integración de la Amazonia transnacional, de la Amazonia sudamericana. Se trata de una nueva escala para pensar y actuar en la Amazonia. Este dato es importante por múltiples razones. Primero, porque la unión de los países amazónicos puede fortalecer el Mercosur y, de cierta manera, construir un contrapunto para las relaciones con el ALCA y con

3 LBA es un vasto proyecto de investigación coordinado por Brasil en asociación con la NASA y, en menor escala la Unión Europea, denominado Large Scala Biosphere-Atmosphere Experiment in the Amazon. Probem fue creado para implantar la investigación y desarrollar el uso de la biodiversidad a través del fortalecimiento de la biotecnología.

4 (Nota de la traducción) Francisco Alves Mendes Filho (1944-1988), conocido como Chico Mendes, asesinado en 1988, fue uno de los más grandes ecosocialistas brasileños, creador de la Alianza de los Pueblos de la Floresta y ganador del Premio Global 500 (ONU, 1987). [Porto Gonçalvez, Carlos Walter (2009) "Chico Mendes, un ecosocialista”. OSAL, vol. X, núm. 25, 151-154.] 
la propia Unión Europea. En segundo lugar, para tener una presencia colectiva y una estrategia común en el escenario internacional, fortaleciendo la voz de América del Sur. En tercer lugar, porque es fundamental para establecer proyectos conjuntos en cuanto al aprovechamiento de la biodiversidad y del agua, incluso en las áreas que ya tienen un equipamiento territorial e intercambio, como es el caso de las ciudades gemelas ubicadas en diferentes puntos de las fronteras políticas.

Además, ese dato es importante porque podría ayudar a contener las actividades ilícitas - narcotráfico, contrabando, lavado de dinero, etc.- y una posible "ayuda" militar en el territorio brasileño. Hay una creciente presencia militar en la fachada del Pacífico y Centroamérica, a través de lo que se denomina "localidades de operaciones avanzadas", cuya mayor expresión es el Plan Colombia. Brasil se convirtió en una isla rodeada de "localidades de operaciones avanzadas" por todos lados, con instalaciones norteamericanas apoyadas por la Unión Europea, con excepción de las fronteras con Venezuela y Argentina. Brasil intentó impedir ese cerco con varias respuestas, como con la creación del Ministerio de Medio Ambiente y el proyecto Sipam (Sistema de Información para la Protección de la Amazonía), aunque cuenta con apoyo financiero para la Policía Federal.

Este proceso llevó a una fuerte reactivación de las fronteras políticas de la Amazonía, consideradas anteriormente como fronteras muertas, y basta ir a Tabatinga y Letícia para constatar la revitalización de las mismas, lo que constituye una preocupación para todos los países.

Pero el hecho de que la globalización incida en la Amazonía a través de la presencia militar en los países vecinos, y en Brasil a través de la cooperación internacional, constituye una diferencia importante.

De hecho, se realiza una articulación sudamericana a través del rescate del Tratado de Cooperación Amazónica (OTCA), y también a partir de la iniciativa de la planificación física de la integración por medio de transporte multimodal, difusión de internet en los países vecinos e intercambio energético. En Roraima se dio el primer paso para la integración oficial a través de la construcción de la carretera que une Manaus con Venezuela. El gas ya es transferido de Bolivia y Perú, y la Bolsa de Mercancías y Estudios propone la extensión de la frontera agropecuaria del centro oeste brasileño hacia los países vecinos.

¿Deseamos que la integración sudamericana según el molde de lo que fue la integración en los años 1970? Nada que decir en contra la expansión de la soja y la ganadería hacia áreas propicias de la sabana, pero sí respecto a esa expansión en áreas de bosques. Este es un desafío para la Ciencia y la Tecnología. La integración debe estar basada en la circulación fluvial, que merece una inversión enorme, pues siempre fue el gran medio de circulación en la Amazonia, y también en la aérea, que fue muy importante y aún lo es para el transporte de cargas de alto valor agregado.

Otro elemento importante de la integración reside en las ciudades gemelas Santa Helena y Pacaraima en Roraima, Tabatinga y Letícia en el Amazonas, además de otras en el Acre, donde ya existen embriones de integración, flujos y equipamientos que podrían acelerar el intercambio. Por otra parte, la ciudad es un elemento fundamental en el desarrollo y planificación de la Amazonía, porque en ella la población está concentrada, constituye el núcleo de las redes de relaciones, y puede incluso impedir la expansión demográfica en el bosque. 


\section{La Amazonia en el espacio nacional: una región en sí}

La segunda hipótesis que propongo se refiere al lugar de la Amazonia en Brasil. Se afirma aquí que la Amazonía no es más mera área de expansión de la frontera móvil, sino una región en sí, sobre la base de dos argumentos: la nueva aparición de la frontera y los avances regionales en términos económicos, sociales y políticos.

La situación de conflicto entre desarrollo y protección ambiental se reflejaba en las políticas públicas de la década de 1990 que eran, a la vez, expresión e inducción del conflicto. Por un lado, el Ministerio de Medio Ambiente implementaba una política de protección de los bosques y, por otro lado, el Ministerio de Planificación y Presupuesto, creaba corredores de exportación. Evidentemente, los corredores de exportación coincidían con los ecológicos.

Así, se multiplicaron las unidades de conservación, se demarcaron tierras indígenas y se creó el proyecto Áreas Protegidas de la Amazonia (Arpa), una iniciativa del Banco Mundial y del WWF para ampliar en un $10 \%$ las áreas protegidas hasta 2010. Se trata de un proyecto preservacionista que a penas por la presión de la ministra Marina Silva, se aceptó aumentar muy poco el área del uso sostenible (noventa mil kilómetros cuadrados). Por lo tanto, la Amazonía tendrá, en breve, más del $30 \%$ de su territorio en áreas protegidas, un área equivalente al territorio de España. ¿Cuál fue el resultado de ese conflicto?

\subsection{Las nuevas facciones de la frontera móvil}

Aquí se plantea una hipótesis polémica: en la década de 1990, el ambientalismo dominó y se plasmó como la tendencia al agotamiento de la Amazonia como frontera móvil, es decir, como frontera de expansión económica y demográfica en el territorio. Los frentes pasaron a presentar diferencias con la expansión de la frontera en la década de 1970, tales como:

1. En los años 1970, lo que sostuvo la frontera fueron los incentivos fiscales y la migración generalizada del país entero, inducida por el gobierno federal. En la actualidad, la migración dominante es intrarregional, de un estado a otro y, sobre todo, rural-urbana (con excepción hecha del Mato Grosso, que continúa atrayendo población de fuera, principalmente del Sur y del Nordeste).

2. Otro elemento importante de diferenciación es el manejo de los frentes por parte de Belém y de Cuiabá, sobre todo, hoy de ámbito regional. Así, lo que hay de nuevo en la expansión de los frentes es que está orientada por madereras, ganaderos y sojeros ya instalados en la región, y que la promueven con recursos propios. No se trata, pues, de una expansión subsidiada por el gobierno federal, como fue la de la frontera en los años 1970.

3. Además, los frentes de hoy están localizados. En los años 1970 se ubicaban en las dos grandes arterias, Belém-Brasília y Brasília-Cuiabá, de modo que la expansión siguió la franja de los bosques. Ahora, los frentes se ubican alrededor de las carreteras que ya existían, las que pretenden ser pavimentadas o las abiertas por los propios madereros y ganaderos. 
A la vuelta del milenio, sin embargo, la frontera tomó nuevo aliento. Son tres los grandes frentes en la Amazonia hoy: una parte de San Felix del Xingu, Sudeste de Pará, hacia el río Iriri; otra parte del extremo norte de Mato Grosso por la carretera Cuiabá-Santarém, en torno a cuya pavimentación hay gran discordia, pues no transcurre por el borde, sino por el medio del bosque; y la tercera parte del Norte de Mato Grosso y de Rondônia hacia el Sur del Estado de Amazonas.

La tecnología sirve también para la destrucción del bosque: los madereros se están apoderando de tierras vía satélite, descubren dónde hay tierras disponibles y ocupan las tierras en inmensas glebas. Un lado tragicómico es que existen, en el Sur del Amazonas, muchos granjeros que vinieron del Pontal do Paranapanema, expulsados por el $\mathrm{MST}^{5}$, porque no tenían tierras regularizadas; sin embargo, el MST sabe muy bien quién posee o no tierras regularizadas.

Pero el elemento más importante que justifica la hipótesis aquí tratada es la consolidación del poblamiento, en contrapartida con los frentes de expansión.

\subsection{La consolidación del poblamiento}

La tendencia a la consolidación del poblamiento es patente en el avance económico significativo y en la tecnificación de la agroindustria en la sabana, particularmente en Mato Grosso, donde se planta soja y ahora también algodón colorido. Con el crecimiento de la producción y el aumento de la productividad de la soja, la tierra ya no es ocupada como reserva de valor, como fue en la época de la frontera anterior. Ahora lo que aparece es el uso productivo de la tierra. En los últimos años, se ha producido un aumento en la ganadería, principalmente en el Sudeste de los estados de Pará y en Mato Grosso, donde se producen mejoras con respecto a los pastos, a los rebaños y a la industria de cuero y de leche; cambios bastante significativos en términos económicos.

Las redes y ciudades permiten la expansión de esa área económica avanzada que se llama "arco de fuego", o de la deforestación o "de tierras degradadas", porque fue donde se expandió la frontera y la deforestación. Pero es hora de cambiar esa denominación, teniendo en cuenta que se trata de la mayor área productora mundial de soja. Río de Janeiro ya fue un pantano, pero hoy no se llama pantano, sino metrópoli. Se sugiere, entonces, un cambio de nombre para área de poblamiento consolidado, porque la denominación de arco del fuego obstaculiza la política pública.

Por lo tanto, existe un gigantesco enfrentamiento entre la expansión de la agroindustria de la soja, la ganadería, y la explotación de la madera con el uso conservacionista ${ }^{6}$ del bosque, defendido por los pequeños productores, por los ambientalistas y por diversas categorías de científicos.

En los últimos años, hubo una reanudación vigorosa de los frentes, en las tres ubicaciones mencionadas, debido a la valorización de la soja en el mercado inter-

5 (Nota de la traducción) Movimiento de los Trabajadores Rurales Sin Tierra, conocido como Movimiento Sin Tierra, o simplemente MST. Consonante Caldart (2001: 207), el movimiento fue creado entre los años 19791984 y formalizado en el Primer Encuentro Nacional de los Trabajadores Sin Tierra, entre el 21 y 24 de enero de 1984. [Caldart, Roseli Salete. (2001) "O MST e a formação dos sem-terra: o movimento social como princípio educativo”. Estudos Avançados, vol. 15, núm. 43, 207-224.]

6 (Nota de la traducción) Ver nota 5. 
nacional y a las incertidumbres de la economía nacional. Se concluye, así, que la frontera es un elemento estructural del crecimiento económico en Brasil, pero hoy depende de la coyuntura; es decir, se expande o se retrae en función de la coyuntura económica y política. Es, por lo tanto, un concepto espaciotemporal. Es muy difícil establecer un pronóstico sobre el conflicto entre agronegocios y conservación del bosque. Un grupo de investigadores del Inpa (Instituto Nacional de Pesquisas de la Amazônia) liderado por un norteamericano realizó un modelo afirmando que en 2020 la Amazonía estaría totalmente destruida. Un modelo lineal, que no prevé alteración alguna, no se puede aceptar en un mundo de imprevisibilidad.

Hoy, la Amazonia ya no es una mera frontera de expansión de fuerzas exógenas nacionales o internacionales, sino una región en el sistema espacial nacional, con estructura productiva propia y múltiples proyectos de diferentes actores. En ella, la sociedad civil pasó a ser un actor fundamental, tanto en el campo como en las ciudades, especialmente por sus reivindicaciones de ciudadanía, que incluso influyen en el desarrollo urbano. El Grupo de Trabajo Amazónico (GTA) ${ }^{7}$ tiene 315 asociaciones, entre ellas la federación de organizaciones indígenas. Los indios son muy expertos, aprenden todo rápidamente, mantienen su cultura y crecen a un ritmo que es el doble de la tasa nacional. Además, crean $\mathrm{ONG}^{\prime}$ s para ayudar a otras comunidades no tan informadas como las suyas.

Otro grupo importante, más localizado, es el de Altamira, antiguo proyecto de colonización en que la pequeña producción está organizada y tiene una fuerza política significativa, resistiendo a la construcción de la hidroeléctrica de Belo Monte.

Actores fundamentales son los gobiernos estatales, que, con la crisis del Estado central, asumieron responsabilidades y fuerza política. Es interesante e importante saber que estos gobiernos, por sus condiciones histórico-geográficas, tienen estrategias diferentes. Mato Grosso y Pará tienen estrategias extensivas de uso de la tierra, el estado de Amazonas tiene una estrategia puntual industrial, ubicada en Manaus; el Acre y el Amapá se basan en la estrategia de la florestania ${ }^{8}$, modernización del extractivismo; en Rondônia se busca expandir la pecuaria e incluso la soja, y, en Roraima, la soja en la sabana, rodeada por bosques y tierras indígenas. El municipio también es un ente político que tiene voz en la región, aunque sin recursos financieros. Económicamente, no tiene fuerza, pero la tiene desde el punto de vista político, y es responsable de la urbanización reciente, transformando las villas en ciudades.

Las empresas del agronegocio, además de las madereras y ganaderos, son otros actores que se están afirmando y expandiendo y no sólo en Mato Grosso. Lo que ha pasado desapercibido, a mi entender, en todos los proyectos y en todas las escalas, es justamente el hecho de que la Amazonia hoy es una región que posee una dinámica propia: tiene veinte millones de habitantes, hay demandas específicas y resis-

7 (Nota de la traducción) Red de entidades de la sociedad civil para el monitoreo de políticas y de conflictos socio ambientales para la sostenibilidad de la biodiversidad amazónica.

8 (Nota de la traducción) Concepto creado por Antonio Alves, cronista, poeta y pensador acreano, a finales de los años 80. El vocablo es una fusión de dos términos en lengua portuguesa: floresta (bosque) + cidadania (ciudadanía). El supuesto de su formulación es la preservación de las riquezas naturales del bosque como condición para el desarrollo humano económico y social. [Pinheiro, Francisco de Moura. (2010) "Florestania - A Cidadania da Floresta Vista a Partir dos Conceitos de Biopolítica, Significantes Vazios e Ecologia dos Saberes". http://www.intercom.org.br/papers/nacionais/2010/resumos/R5-2515-1.] 
tencias organizadas y, una estructura productiva propia, lo que comprueba su cambio de condición, incluso con una nueva geografía. En ella reconozco tres macrorregiones (Figura 3): la primera es aquella que llaman "arco de fuego" y que denomino arco del poblamiento consolidado, porque es donde están las ciudades, las mayores densidades demográficas, las carreteras y el núcleo de la economía; la otra macrorregión, la Amazonia central, corresponde al resto del estado de Pará, que es la porción más vulnerable de la Amazonia, porque está atravesada por los ejes, por las carreteras y es donde están dos de los frentes localizados; la última es la Amazonia occidental, que tiene la mayor área de frontera política y es la más preservada (porque no fue cortada por carreteras y su poblamiento fue puntual en la Zona Franca de Manaus, mientras el resto del estado quedó abandonado). Y el hecho de ser una región en sí constituye una fuerza de resistencia a la destrucción del bosque.

\section{Para terminar: ¿Cómo impedir la destrucción de los bosques? El papel de las políticas públicas}

Si la Amazonía es efectivamente una región, entonces hay que sustituir la política de ocupación por una política de consolidación del desarrollo. Una política de ocupación no tiene cabida, porque la región ya está ocupada. Los bosques que quedan deben permanecer para sus habitantes. Es necesario articular los diferentes proyectos y los diversos intereses y conflictos que inciden en la región. El gobierno actual pretende ser un hito en el rumbo del desarrollo regional. Elaboró un nuevo Plan Amazonia Sostenible (PAS), con el que pretende superar la polaridad conflictiva entre la política ambiental y la de desarrollo.

El gobierno actual propone también en el PPA (Plan Plurianual 2004-2007) la necesidad tanto de productividad y competitividad como de inclusión social, empleo y renta. En el caso de la Amazonia, existe un nuevo principio, el de la transversalidad, en el que el medio ambiente deja de ser tratado como una variable independiente y que está presente en las políticas de todos los ministerios. Importantes también son los cinco ejes estratégicos del PAS, que van a ser debatidos con la sociedad: gestión ambiental y ordenación del territorio, nuevo patrón de financiación, inclusión social, infraestructura para el desarrollo y producción sostenible con innovación tecnológica y competitividad.

El punto central, que genera conflictos, es la cuestión de la pavimentación de la carretera Cuiabá-Santarém (BR-163), porque, por un lado, las corporaciones de la soja presionan al gobierno para una pavimentación rápida, ya que es considerada elemento central para el flujo de la producción por el Norte, con el objetivo de acortar distancias y bajar costos. Por otro lado, los ambientalistas y los pequeños productores no quieren la pavimentación. 


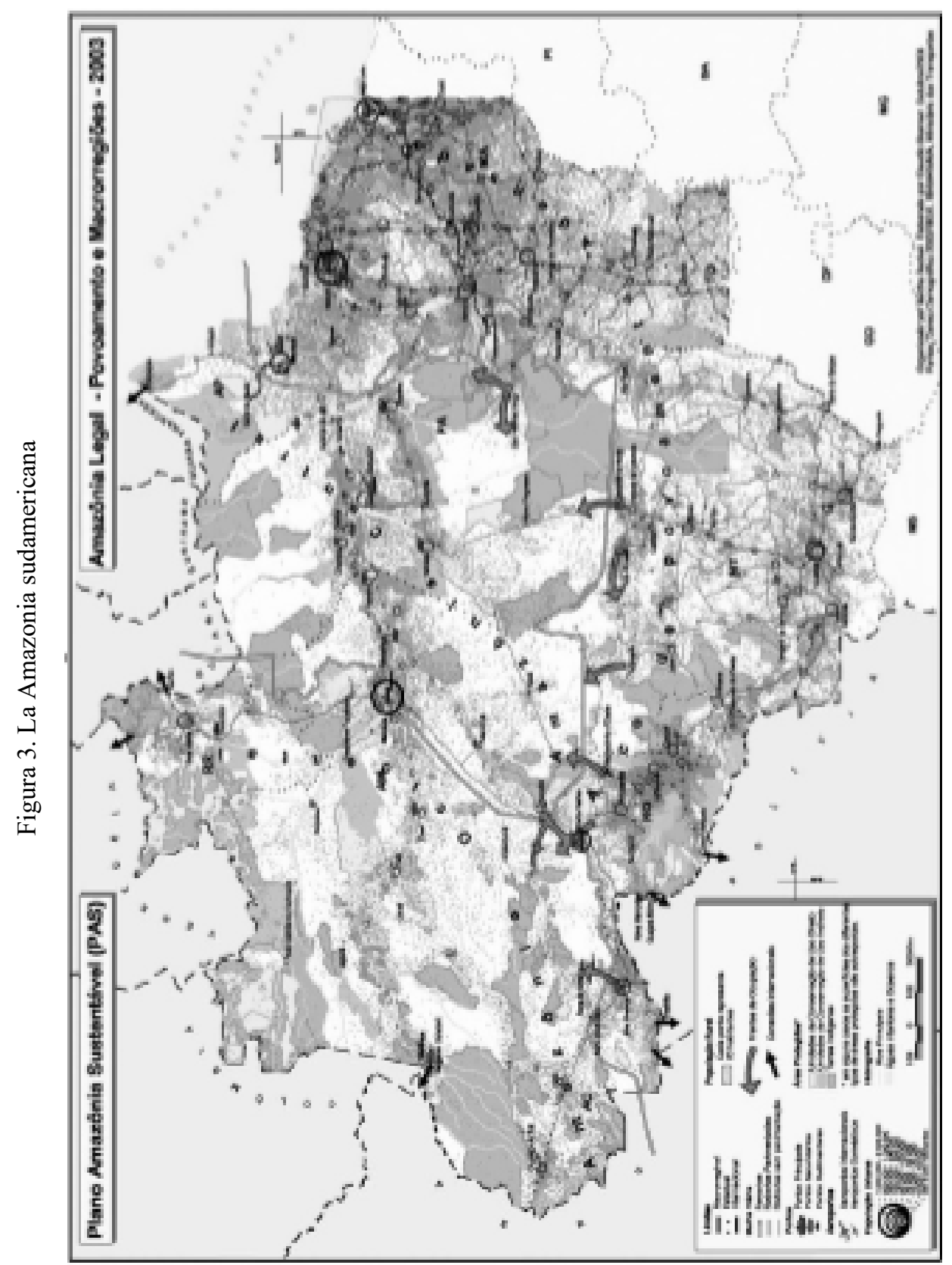

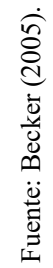


El gobierno propuso que se hiciera un modelo para transformar la carretera Cuiabá-Santarém en una carretera inductora del desarrollo, en vez de una inductora de depredación. Es importante señalar que en Brasilia se crearon grupos de trabajo interministeriales para los planes gubernamentales, algo extremadamente positivo, donde catorce ministerios participan en la nueva planificación de la carretera como instrumento de desarrollo.

El Incra (Instituto Nacional de Colonización y Reforma Agraria) está haciendo un esfuerzo para preparar un catastro basado en la centralidad de la cuestión de la tierra. En vez de dar títulos de tierra, se podrían hacer concesiones de tierras condicionadas a determinados comportamientos. El problema es que todos los actores en la Amazonia (ganaderos, madereros, indios, pequeños productores) tienen, como primera demanda, la presencia del Estado, por diferentes motivaciones. Como segunda demanda desean la zonificación. Estas demandas expresan, por un lado, "la necesidad de una definición clara de las reglas del juego", es decir, del fortalecimiento institucional y, por otro, la pertinencia de la subrregionalización, porque las regiones tienen finalidades propias y problemas específicos. El Estado puede dialogar mejor con estas necesidades específicas, encontrar las interlocuciones necesarias y orientar mejor los recursos para atenderlas.

Finalmente, pero no menos importante, a la par que el fortalecimiento institucional y la regionalización, corresponde a la Ciencia, Tecnología e Innovación un papel primordial en la sostenibilidad de los ecosistemas forestales, por su importancia económica, social y política.

El bosque sólo dejará de ser destruido si tiene valor económico para competir con la madera, con la ganadería y con la soja. Incluso con los grandes avances en su protección, la cuestión de mantener la capacidad forestal sostenible todavía no ha sido solucionada. Los bosques y las tierras son bienes públicos y, por eso, son recursos que están bajo el poder del Estado, que tiene autoridad para disponer de ellos, según el interés de la nación. Se propone, así, una verdadera revolución científica para la Amazonia Forestal.

En Brasil ya hubo tres grandes revoluciones tecnológicas: la explotación del petróleo en aguas profundas; la transformación de caña de azúcar en combustible (alcohol) en la Mata Atlántica y la corrección de los suelos de la sabana, que permitió la expansión de la soja. Es hora de implementar una revolución científica en la Amazonia que establezca cadenas tecno-productivas con base en la biodiversidad, desde las comunidades del bosque hasta los centros de la tecnología avanzada. Este es un desafío fundamental hoy, que será aún mayor con la integración de la Amazonia sudamericana..

\section{Bibliografía}

Becker, Bertha K. (1999) "Escenarios a corto plazo para el desarrollo de la Amazonia". Cadernos NADIAM, Brasília: MMA.

Becker, Bertha K. (2004) Amazonia: geopolítica en la vuelta del III milenio. Rio de Janeiro: Garamond.

Boulding, Keneth (1966) "The Economics of Coming Space-ship Earth" en: Environment Quality in a Growing Economy. Baltimore: John Hopkins. 
IIRSA (2002) Iniciativa para implantación de la infraestructura regional de América del Sur. Brasilia: Secretaría de Planificación e Inversiones Estratégicas.

Polanyi, Karl (1980) La gran transformación. Rio de Janeiro: Campus. 